\title{
Research on the influence of college students' crowd decision- making on improving the content of public account tweets
}

Xiangpeng Yang and Yi He

Central University of Finance and Economics, Beijing, China

\begin{abstract}
Purpose - As human beings step into the age of information network, big data technology is constantly improving the intelligence level of various agents such as individuals and enterprises. The crowd decisionmaking of the intellectual community plays an important role in the active participation of many individuals and schools in giving their wisdom, effectively solve the problems of negative internet communication, single publicity media and unprofessional promotion team in WeChat public account.

Design/methodology/approach - This paper aims to optimize the content and improve the effectiveness of network ideological and political education in universities. This study analyzes five highly popular WeChat public accounts at the Central University of Finance and Economics in 2019. It obtains the popularity index of tweets using the WeChat communication index algorithm and finds that the important factors that influence tweet popularity are release time and content value.

Findings - To improve the public account tweets, this study highlights the connection between the tweets' value and students' emotional needs, which enhances the value of tweet content in students' life and provides more original and distinctive content.

Originality/value - This study found that the content and interest of college students are tweet time, tweet value and tweet content. Therefore, the public account of college ideological and political education should be improved from the following three aspects: realizing the connection between the value of tweet content and students' emotional needs; enhancing the value of tweet content in students' life and learning; and insisting on the original and distinctive original intention of tweet content.
\end{abstract}

Keywords Optimize, Official account tweet content, Suggestions

Paper type Case study

(C) Xiangpeng Yang and Yi He. Published in International Journal of Crowd Science. Published by Emerald Publishing Limited. This article is published under the Creative Commons Attribution (CC BY 4.0) licence. Anyone may reproduce, distribute, translate and create derivative works of this article (for both commercial and non-commercial purposes), subject to full attribution to the original publication and authors. The full terms of this licence may be seen at http://creativecommons.org/ licences/by/4.0/legalcode

This paper is a phased achievement of the key project of the Beijing Social Science Foundation, "Research on collaborative development of e-commerce in Beijing, Tianjin, and Hebei" (No. 15JDJGD093); a special project of ideological and political education for college students in Beijing "Research on content optimization of network ideological and political education in universities based on data mining" (No. BJSZ20182X41); Beijing Outstanding Young Scientist Program (BJJWZYJH01201910034034); supported by the 111 Project (B20094); the Program for First-class Discipline Construction in Central University of Finance and Economics; the Beijing Program for "Double First-Class" Construction Project of Central Universities.
Received 14 October 2020 Revised 3 November 2020 Accepted 5 November 2020 


\section{IJCS \\ 5,1}

In nature and human society, there are numerous group collaborations, such as bird flocking, ant colony. Scientists call these collaborations the "swarm intelligence." The group collaborations in the information and networking era are influenced by scales, the nature of the group intelligence and group behaviors. We call this more complex group intelligence "crowd intelligence." In the phenomenon of crowd intelligence, crowd decision-making plays an important role in mobilizing many individuals and schools to respond positively and actively participate in giving their opinions and wisdom.

However, recent years have seen some problems involving the WeChat public accounts of these college and university organizations, such as negative internet communication, single media and not professional promotion team. Most WeChat official accounts push content in plain text or combination of pictures and texts, whereas only a few universities try to adopt new methods such as audio and video (Cao, 2018). And most official accounts are lack of public opinion monitoring management institutions, and the team strength is insufficient (Yu, 2017).

Therefore, for data availability and timely analysis, this study chose five popular WeChat public accounts from 2019 (CUFE, What CUFE, CUFE Bengdou, CUFE Student Affairs Office and CUFE Graduate Student Union) as research objects. The popularity index of tweets was obtained through WeChat communication index (WCI) algorithm, and the important factors affecting tweet popularity were identified and examined, which helped map the content and interest points of college students. Finally, school sorts out the content and interest points that students pay attention to and puts forward countermeasures and suggestions to improve the content of the tweets of the public account through cooperative methods such as adding, deleting, modifying and supplementing.

\section{Development status of and problems encountered by WeChat public accounts of ideological and political organizations in colleges and universities} Since the emergence of the WCI in 2014, the WeChat public accounts of universities have entered a ranking competition stage from a bulletin board stage. The WeChat public accounts of universities in China have exceeded 60,000, and its active status has surpassed Weibo to become the communication window with currently the highest utilization rate and the best feedback effect for universities. The Central University of Finance and Economics (CUFE) set up a new media matrix that focuses on enhancing teacher-student interaction, knowledge sharing, data analysis and market competition awareness. The WeChat public accounts of online ideological and political organizations in colleges and universities currently have three deficiencies, as explained next.

\subsection{Internet has affected college students more negatively}

The network is an open communication platform with a high speed and wide range of information transmission. This open and high-speed information transmission also adds the characteristics of diversification and complexity to the information content. However, much of this information do not necessarily conform to the actual situation, with some even false and untrue, which significantly shapes the development of ideological and political education in colleges and universities. For example, there has been a high number of college students addicted to online games, which directly affects their studies and delays their development. So many negative effects of the internet severely complicate ideological and political education in colleges and universities. 

which have relied on traditional network carriers for many years. Many websites are slow to update, which reduces the communication information of the QQ WeChat group. As society develops, college students have become more active in WeChat public accounts, Douyin and other new social media networks. However, officials of colleges and universities have failed to keep up with the times; thus, they have clearly fallen behind in the network frontier of college students and in the expansion of multiple work carriers.

\subsection{Promotion team of network ideological and political education in universities is not professional}

While college students are extremely active in the network, their teachers' degree of familiarity with the network, breadth of application and use of the network are lagging. Many college students hold bad values and indulge in online network games. They would rather accept network solutions without scientific demonstrations or authoritative explanations, even indulging in watching foreign cartoons in Stations B and D. Their values virtually deviate from those of mainstream society. In the face of these difficulties, there is no professional team to guide online ideological and political education in colleges and universities.

\section{Data sources and analysis methods}

\subsection{Data sources}

To improve the availability and validity of data, the data source of this paper consists of 3,189 articles pushed by five popular public WeChat accounts at the CUFE in 2019. These are the five most influential public accounts with the most subscriptions. These five public accounts are more representative, both official and unofficial, both graduate and undergraduate students. The five popular public WeChat platforms are "CUFE," "What CUFE," "CUFE Bengdou," "CUFE Student Affairs Office" and "CUFE Graduate Student Union."

\subsection{Analytical methods}

In this paper, the most authoritative index in evaluating the influence of WeChat in China, the WCI, serves as the research parameter. According to the setting of the WCI algorithm, the attention to WeChat content is reported in the reading index, WCI $(\mathrm{R})$; the response rate is represented in the thumbs-up index, $\mathrm{WCI}(\mathrm{Z})$; and the WCI calculated by weight reflects the "heat." The attention and response rate of college students to the content of network education are important measures of the effectiveness of network education (Teresi Jeanne A,2016). In the WCI algorithm, attention mainly refers to how often people read an article, which is used to measure the coverage of information transmission (Clare Tyrer, 2019). The response rate mainly concerns the interaction between fans and public WeChat accounts after reading the article. The WCI heat calculated by combining the two can more comprehensively reflect the content and interest points to which college students pay attention (Huang et al., 2019). The specific indicators and calculation formulas are shown in Table 1. 


\section{IJCS \\ 5,1}

76

Table 1.

Reading index and the thumbs-up

formula

\begin{tabular}{llcl}
\hline Level 1 indicators & Secondary indicators & Weight $(\%)$ & Standardized method \\
\hline Reading index & Average daily reading (R/d) & 40 & $\ln (\mathrm{R} / \mathrm{d}+1)$ \\
$(80 \%)$ & Average number of readings (R/n) & 45 & $\ln (\mathrm{R} / \mathrm{n}+1)$ \\
& Maximum number of readings (Rmax) & 15 & $\ln (\mathrm{Rmax}+1)$ \\
Thumbs-up index & Average thumb up number per day (Z/d) & 40 & $\ln (10 * \mathrm{Z} / \mathrm{d}+1)$ \\
$(20 \%)$ & Post-average thumbs-up number (Z/n) & 45 & $\ln (10 * \mathrm{Z} / \mathrm{n}+1)$ \\
& Highest number of likes (Zmax) & 15 & $\ln (10 * \mathrm{Zmax}+1)$
\end{tabular}

\section{Overall description of WeChat tweets and analysis of influencing factors}

\subsection{General description of WeChat tweets}

We analyzed 3,189 tweets posted by the five most popular WeChat public accounts of the CUFE in 2019, divided into seven categories: mainstream values education; learning and employment; campus culture; life service; news reporting; and notification and voting and investigation. We used the total reading volume $(\mathrm{R})$ of tweets to evaluate their effective reception and the thumbs-up rate $(\mathrm{Z} / \mathrm{R})$ to represent readers' acceptance of these tweets.

Statistics show that these five accounts maintained a high level of activity throughout 2019. As official WeChat platforms, the publication volumes of the CUFE Graduate Student Union, CUFE, CUFE Student Affairs Office, and What CUFE remained high year-round. While CUFE Bengdou is an unofficial account, its publication volume is higher than each of the other four official accounts, which may be attributed to its focus on marketization. Overall, two public CUFE accounts, What CUFE and CUFE Bengdou, had almost all highly read tweets. This may be explained either by the appeal of the tweets themselves or by the number of readers following them. In terms of tweet content and form, the abovementioned seven categories all adopt various forms of expression, such as pictures, audio, video and text. This means that readers prefer a rich variety of tweets, with text-only tweets not being attractive enough. In terms of tweet content, those pertaining to campus culture, learning and employment, and news reports were the top three. Students were most interested in tweets about school development, campus life, campus culture and others, which they find highly relevant to themselves. In addition, aside from focusing on seeking employment information, college students also go to WeChat as the main channel for general information, as evident in news reports being ranked third.

\subsection{Influencing factors of WeChat tweets}

Reading and thumbs-up rates are the basic indicators of the reach of public accounts or tweets. Based on our analysis of number of tweets read, thumbs-up rates and the association between posting time and number of tweets read, we found that the popularity of public tweets is shaped by several factors.

3.2.1 Tweet release time. Our analysis indicates that the publishing time of tweets from official accounts had a direct and close correlation with tweet popularity. Despite some data showing that students' WeChat reading time conflicts with their class time, this finding 
highlights the real-time nature of tweets. If class time is not taken into account and the data analysis considered in isolation, then 9:00, 12:00 and 14:00 are optimal times for posting tweets, as shown in Figure 1.

3.2.2 Values of tweets. The value orientation of information is based on a society's values and beliefs. Because of its emphasis on common interests and values, it plays a significant role in guidance and enlightenment. When the values and emotional orientation of information are consistent, communication can become more effective and efficient. Research has shown that mainstream value articles account for a large proportion of highpoint-rate tweets released by the CUFE's public accounts. This indicates that the values conveyed by these tweets match the emotional needs of students, demonstrating high communication effectiveness and efficiency.

3.2.3 Contents of tweets. Figure 2 shows that tweets with the highest overall reading volume and thumbs-up rates are about campus culture, news items and study and employment. Tweets that are highly relevant to students' daily and academic life are more likely to affect them psychologically and emotionally. A common feature of these tweets is their high originality and sophistication. Generally speaking, they are all original tweets; the so-called boutique original tweet is characterized by positive energy, true feelings and a simple style. These are coupled with strict theme planning,

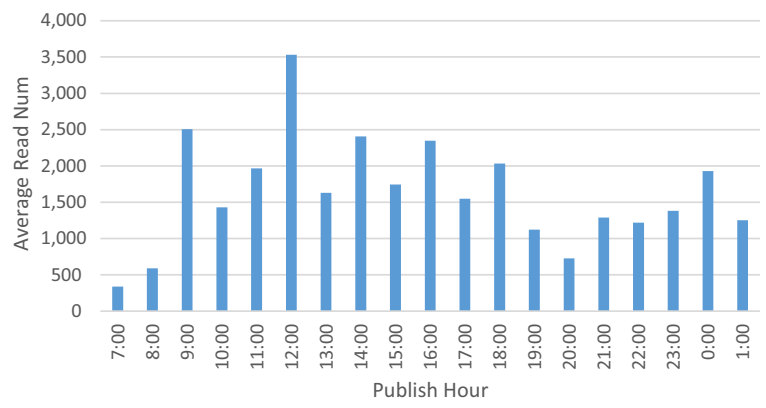

Top 50 thumbs-up structure of tweet content type

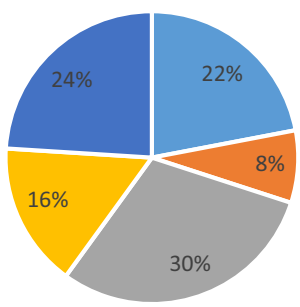

\begin{tabular}{|c|c|}
\hline $\begin{array}{l}\text { campus culture } \\
\text { life service }\end{array}$ & $\begin{array}{l}\text { study and employment }=\text { news stories } \\
\text { mainstream value }\end{array}$ \\
\hline
\end{tabular}

Public account tweets

Figure 1. Average read number vs publication hour
Figure 2. Structure chart of tweet content type (top 50 thumbs-up) 
copywriting design and collection and editing processes that use rich materials from university campuses and are familiar to college students. This kind of tweet communicates effectively and has great value in shaping young students' thoughts. For example, tweets with the highest thumbs-up rate introduce college students to people and events through pictures and text. This category receives the most attention main because its subjects are real people and discuss topics relevant to students' daily and academic life, which can call students to action. This induces a strong emotional resonance and value identification among readers, prompting them to respond with a thumbs-up.

\section{Suggestions to optimize the tweet content of official accounts}

\subsection{Emphasize the connection between the tweets' content value and students' emotional needs}

Student-centered thinking should be established in the use of public accounts in colleges and universities. First, we should pay attention to the emotional aspect of tweets. The higher their emotional impact, the more attractive they will be. Second, to adapt to the psychology of college students, tweets must show "intuition" and "sensibility" for easier acceptance. Third, tweets should find the audiences' "pain point," "itch point" and "excitement point" to ensure that they grab readers' attention and gain their favor, thus forming a loyal reader group. Although new media products do not need grand-narrative ability, they should be nevertheless relevant to college students and pique their interest. Even content that reflects mainstream values should be familiar to college students. New media products can become "hot" if they can make college students feel "carefree." Upon encountering tweets they agree with, college students can repost them in their respective circles of friends to achieve secondary dissemination, allowing certain information to spread exponentially within the relevant groups and further extend their influence.

\subsection{Enhance the value of tweet content in students' life and learning}

The factors contributing to identity and sharing among college students include the interest, value and emotion of tweets. Meeting students' practical needs is key. When establishing WeChat public accounts and creating tweets, education in the spirit of competition, adherence to the law, teamwork and responsibility should be introduced promptly. The aim here is to enhance students' ability to adapt to society and survive and improve their ability to analyze and solve problems, express, strive and innovate. Simply put, network ideological and political education should endeavor to educate students on how to live, survive and behave.

\subsection{Adherence to the original content and style of the public account}

WeChat public accounts are important vehicles of ideological and political education in universities. In the process of constructing and operating them, everything must be based on reality. Considering the school's culture, WeChat accounts can produce tweets that meet students' actual needs and school characteristics. Not only does this help ensure a fit between school culture and students' needs but it is also conducive to the spread of WeChat, maximizing the role of ideological and political education networks on the platform. For example, tweets must adhere to research first and reflect students' learning life and school culture. Moreover, diverse and popular forms of tweets should be created according to ideological and political education 
themes. Finally, these tweets can encourage students to contribute actively in school, facilitating brainstorming.

\section{Conclusion}

The crowd decision-making of the intelligent group plays an important role in the active participation of many individuals and schools in giving their opinions and wisdom and effectively solves the problems of negative internet communication, single publicity media and unprofessional promotion team in WeChat public account of universities. In this paper, WeChat propagation index WCI algorithm is used to analyze five popular WeChat public accounts of Central University of Finance and Economics in 2019. It is found that the content and interest of college students are tweet time, tweet value and tweet content. Therefore, the public account of college ideological and political education should be improved from the following three aspects: realizing the connection between the value of tweet content and students' emotional needs, enhancing the value of tweet content in students' life and learning and insisting on the original and distinctive original intention of tweet content.

\section{References}

Cao, Q. (2018), "Research on innovation of ideological and political education by using WeChat public account in colleges and universities - a case study of Henan Universities", Journal of Zhongyuan Institute of Technology, Vol. 29 No. 5, pp. 45-48, 65.

Clare Tyrer (2019), "Beyond social chit chat? Analysing the social practice of a mobile messaging service on a higher education teacher development course", International Journal of Educational Technology in Higher Education, Vol. 16 No. 1.

Huang, L., Mou, J., Eric, W.K. See-To and Kim, J. (2019), “Consumer perceived value preferences for mobile marketing in China: a mixed method approach", Journal of Retailing and Consumer Services, Vol. 48.

\section{Further reading}

Blais-McPherson, M. and Rudiak-Gould, P. (2016), "Strengthening inter-disciplinary and interideological collaboration on REDD: a cultural theory approach", Global Environmental Change, Vol. 42, p. 42.

Cecilia Gimenez, M., Paz García, A.P., Burgos Paci, M.A., et al. (2016), "Range of interaction in an opinion evolution model of ideological self-positioning: contagion, hesitance and polarization", Physica A: Statistical Mechanics and Its Applications, Vol. 447, p. 447.

Crasnow, S. (2017), "Process tracing in political science: what's the story?", Studies in History and Philosophy of Science.

Cristian, C., Encarnação, S. and Pinheiro, F.L. (2019), "The higher education space: connecting degree programs from individuals' choices”, EPJ Data Science, Vol. 8 No. 1, doi: 10.1140/ epjds/s13688-019-0218-4.

Huang, G. and Zhang, Y. (2015), "Content marketing strategy of WeChat public account: an analysis based on WeChat communication index WCI", Library Journal, p. 9.

Jing, F. and Wei, L. (2016), "A study on influential factors of wechat public accounts information transmission hotness", Journal of Intelligence, Vol. 35, pp. 157-162.

Li, J. (2017), "Establishment and application of New Communication Power Evaluation Model for University WeChat Subscription Number", News Research Guide, 2017, p. 12.

Liu, J. (2017), "Four focal points of carrying out college students' ideological and political education through WeChat public platform", Research on Ideological Education, Vol. 12. 
IJCS

5,1

Ma, L. (2019), "Research on distance education image correction based on digital image processing technology", EURASIP Journal on Image and Video Processing, Vol. 2019 No. 1, doi: 10.1186/s13640-019-0416-9.

Wu, Z. and Tang Zhenhua, L. (2015), "Research on influencing factors of WeChat public account information dissemination", Journal of Intelligence, Vol. 4.

Zhang, W. and Zhang, P. (2017), "Discussion on university students' information acceptance, identification differences and causes of WeChat public Account", Modern Communication, p. 12.

\section{Corresponding author}

Yi He can be contacted at: hezhexing@163.com

For instructions on how to order reprints of this article, please visit our website: www.emeraldgrouppublishing.com/licensing/reprints.htm

Or contact us for further details: permissions@emeraldinsight.com 